\title{
Preparación de muestras para análisis por difracción de rayos $X$ con el equipo Philips PW 1700
}

JOSE LUIS SAGRERA

Dr. en Ciencias Químicas y Ledo. en Ciencias de la Información

CSIC/IETCC

\section{$R E S U M E N$}

En el presente articulo se relacionan las modificaciones necesarias para el caso, muy corriente, de contar con poca cantidad de muestra, asimismo se relacionan las modificaciones dimensionales de algunas partes de la prensa HERZOG que posibilitan con total seguridad el prensado de muestras en polvo para utilizar el cambiador automático y el aditamento rotatorio de las mismas.

Finalmente se da una marcha, paso a paso, para la obtención de muestras prensadas.

\section{$S U M M A R Y$}

In the present article the needed modifications for the very usual case of having a little quantity of samples are related. There are related in the same way the dimensional modifications of some of the pieces of the HERZOG press which allow's with a full security the pressing of dust samples for the use of the automatic adapter and its rotary addition.

Finally it starts out, step by step, for the obtention of pressed samples.

El primer problema en el análisis de muestras en polvo aplicando la técnica de difracción de rayos $\mathrm{X}$ estriba, como en la mayoría de las técnicas analíticas instrumentales, en la preparación de la muestra.

El Instituto Eduardo Torroja ha resuelto el problema de la preparación con menor cantidad de muestra, disminuyendo el diámetro interior del anillo dentro del cual se deposita la muestra en polvo para ser comprimida. El diámetro original del anillo es de $27 \mathrm{~mm}$, siendo la dimensión modificada de $20 \mathrm{~mm}$. Aun con esta reducción del diámetro, la superficie irradiada, utilizando rendija automática de divergencia, cae dentro del diámetro modificado.

El cambiador automático de muestras, PW 1780, tiene un cargador que puede contener hasta 42 muestras. La introducción de dicho cargador en el cambiador automático ha de hacerse inclinando el propio cargador un ángulo sobre la vertical de $45^{\circ}$, lo que supone que los anillos portamuestras se inclinen el mismo ángulo con el posible desplazamiento de los granos de muestra en polvo y la pérdida de horizontalidad de la muestra sobre su anillo correspondiente. Ello lleva a la obtención de un diagrama falso de la muestra en polvo. Este problema se resuelve si la muestra en polvo ha sido preparada en su portamuestras mediante una prensa idónea que compacta el polvo y le dé la consistencia suficiente. no permitiendo que se desplace sobre el anillo del portamuestras.

La prensa automática HERZOG tipo HDIFP, fabricada en 1982, puede prensar la mucstra en estado pulverulento con granulometria comprendida entre 5 y $10 \mu$.

La reducción del diametro interior del anillo original Philips, al anillo modificado en el Instituto Eduardo Torroja, lleva consigo una reducción del diámetro del pistón que actúa sobre la muestra 
en polvo contenida en el anillo. Esta reducción del diámetro también supone la consiguiente reducción en la placa circular de conducción del pistón sobre la muestra en polvo que ha sido depositada en el anillo para ser prensada. Ambas modificaciones, la del pistón y la de la placa circular, se han hecho en nuestro laboratorio construyendo un nuevo pistón e introduciendo un anillo reductor del diámetro en la placa circular, fabricado en bronce.

La prensa HERZOG, antes citada, se alimenta mediante aire comprimido en una bala cilindrica. La presión de trabajo, según manómetro de la propia prensa HERZOG, debe ser de SEIS BAR equivalente a SEIS PSI.

El tiempo durante el cual se ejerce la presión puede fijarse mediante el mando situado en la parte frontal inferior derecha de la prensa; experimentalmente se ha comprobado que produce buen resultado un tiempo de CINCO segundos.

La compacidad de la muestra no se consigue haciendo que actúe el pistón directamente sobre la misma. Este problema ha sido resuelto en nuestro laboratorio utilizando un cilindro macizo de acero con el que se aplana el polvo antes de ser comprimido. A continuación, sobre la muestra plana se coloca una pieza de goma circular obtenida al cortar de un tapón de goma dura una loncha de $3 \mathrm{~mm}$ paralela a las bases del tapón. Sobre el conjunto anillo, muestra y goma se deja actuar al pistón modificado, obteniéndose una muestra compacta que al ser invertida no se deshace, pudiendo introducirse en el cargador del cambiador automático.

Mediante este tipo de preparación puede utilizarse el SPINNER - aditamento que produce la rotación de la muestra durante su análisis-.

Dada la pequeña cantidad de muestra necesaria utilizando los anillos modificados, el obtener particulas de un tamaño menor de 10 micras puede conseguirse, con poco trabajo, porfirizando la muestra en mortero de ágata. La homogeneidad de la misma se consigue en un pequeño molino vibrador de laboratorio.

La reproducibilidad de datos obtenidos, en un análisis cuantitativo, hace necesario el uso de una preparación de muestra mediante prensa. Dado que la presión y el tiempo pueden regularse en la prensa HERZOG, sólo queda fijar la cantidad de muestra a comprimir, factor éste que puede ser fácilmente controlado midiendo volumen de muestra o peso de la misma.

El mantenimiento de la prensa HERZOG utilizada en estos ensayos es fácil, ya que como toda prensa sólo necesita el aceite que transmite la presión al pistón. Dicha firma recomienda los siguientes tipos y marcas:

\section{ARAL VITAM GF-46; BP ENERGOL HLP-D-46; CASTROL HYSPIN AWS-46; CHEVRON EP HYDR. OIL-46; NUTO H-46; MOBIL DTE-25 y TEXACO RANDO OIL HD B-46.}

Los pasos necesarios para la preparación de una muestra, partiendo de polvo homogéneo y con finura comprendida entre 5 y 10 micras, son los siguientes:

1. Colocar el anillo en posición invertida sobre la platina circular de la prensa.

2. Colocar la placa circular sobre el anillo haciendo coincidir su saliente con el orificio de la placa circular.

3. Introducir la muestra en el anillo por el orificio central (bronce) de la placa circular. 
4. Aplanar la muestra mediante el cilindro macizo de acero (Foto 2).

5. Introducir en el centro y encima de la muestra la pequeña loncha de goma dura (Foto 3 ).

6. Comprimir la muestra a 6 bar durante 5 seg. (mando 1 y 2 de la foto 1 ).

7. Quitar verticalmente la placa circular.

8. Retirar el anillo con muestra prensada y loncha de goma dura, sobre una hoja de papel limpio.

9. Invertir el conjunto para que caiga la loncha de goma dura y el resto de muestra no prensada.

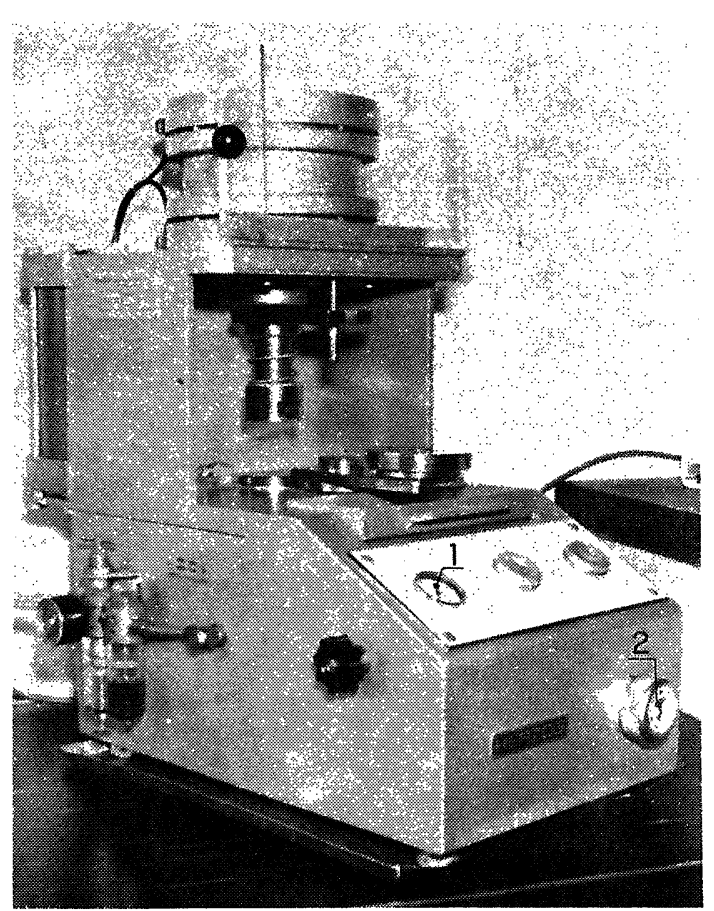

$1 \cdot 1101$

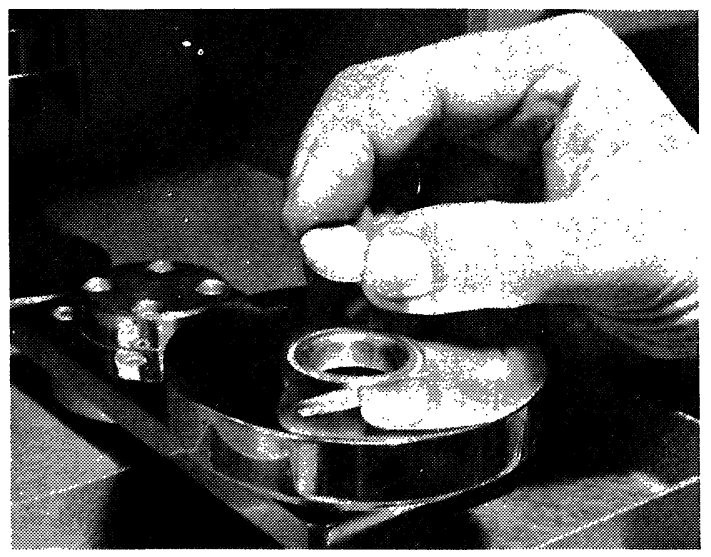

I. oto 3
10. Mediante una cuchilla raspar con cuidado los restos de muestra que sobresalgan del anillo (Foto 4).

11. Colocar de nuevo el anillo con la muestra prensada en la platina circular de la prensa

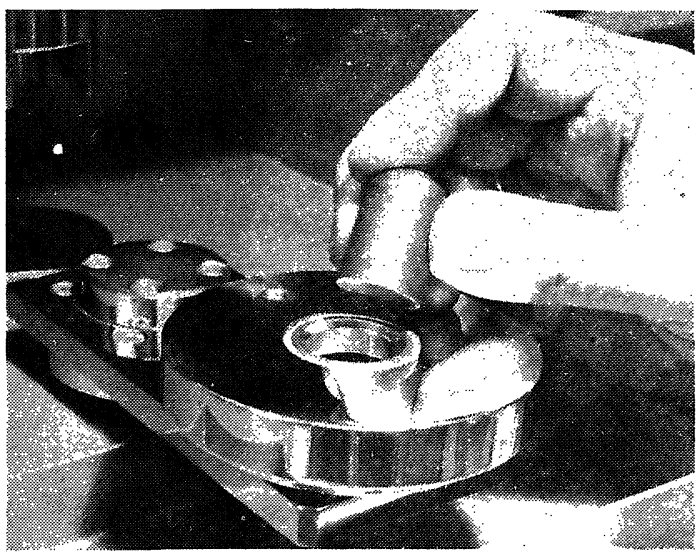

Foto 2

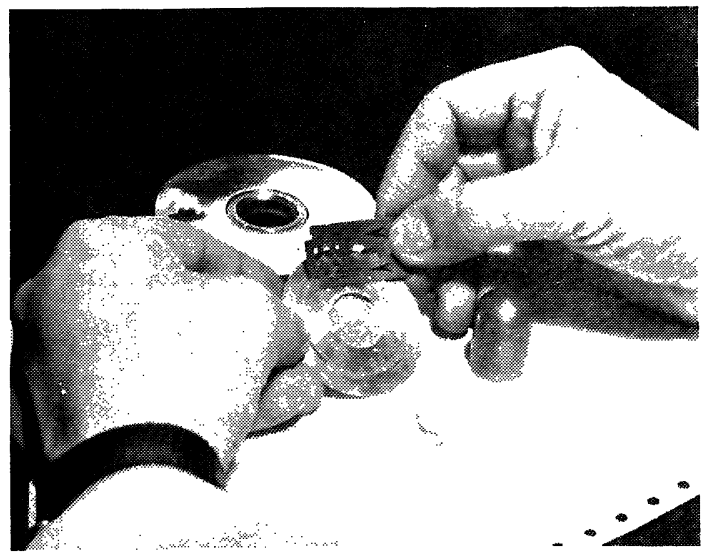

Foto 4 
de manera que la parte a irradiar por rayos $\mathrm{X}$ quede hacia abajo (de igual manera que se colocó para prensar la muestra).

12. Encajar el soporte del portamuestras en el anillo mediante los flejes rectos que éste tiene.

13. Colocar el conjunto anillo-base portamuestras en el cargador del cambiador automático.

En la tabla siguiente se encuentran las medidas originales y modificadas de cada aditamento rectificado.

\begin{tabular}{|c|c|c|}
\hline ADITAMENTO & $\begin{array}{c}\text { Medidas } \\
\text { originales } \\
\text { (mm) }\end{array}$ & $\begin{array}{c}\text { Medidas } \\
\text { modificadas } \\
\text { (mm) }\end{array}$ \\
\hline - Diámetro piston & 27.0 & 19.5 \\
$-\begin{array}{l}\text { Diámetro placa } \\
\text { circular }\end{array}$ & 27.8 & 20.4 \\
- Diámetro anillo & 27.0 & 20.0 \\
$-\begin{array}{l}\text { Diámetro cilindro } \\
\text { macizo acero } \\
\text { Diámetro loncha } \\
\text { goma dura } \\
\begin{array}{l}\text { Espesor loncha } \\
\text { goma dura }\end{array}\end{array}$ & - & 19.8 \\
\hline
\end{tabular}

\section{publicación del i.e.t.c.c.}

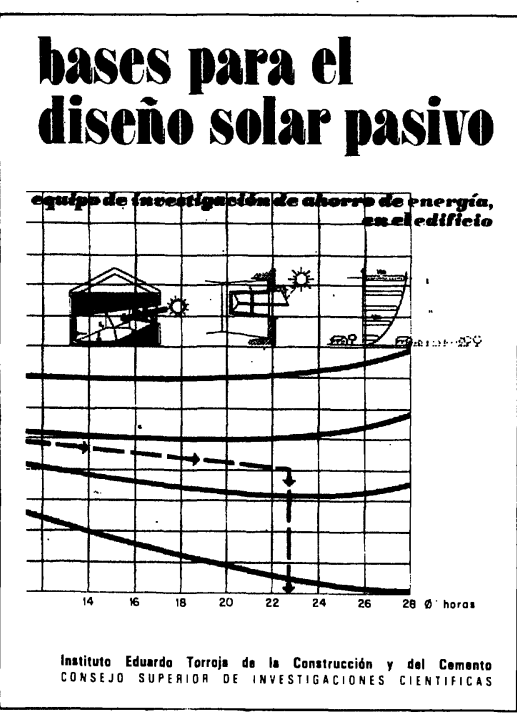

54
Equipo de Ahorro de Energía en el edificio

Dirección y coordinación:

Arturo Garcia Arroyo

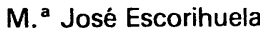

José Luis Esteban

José Miguel Frutos

Manuel Olaya

Bernardo Torroja
Las dificultades de suministro y el alto coste de los productos energéticos convencionales han despertado la atención de los usuarios técnicos $\mathrm{e}$ industriales de la edificación hacia los procedimientos y sistemas en que se basa el aprovechamiento de otras fuentes alternativas de energia principalmente la solar. to solar. Esto ha generado un rapido desarrollo industrial $y$ comercial que, en opinión de los autores de este libro, arrastran los siguientes defectos: un mimético tecnologismo respecto de los sistemas convencionales que violenta las peculiaridades de la energía solar (baja densidad y variabilidad en el tiempol, y una escasa selectividad en la aplicación de los sistemas y procedimientos pasivos dando origen a un ecumenismo arquitectónico solar, al margen de las condiciones climáticas y funcionales específicas de cada caso y lugar.

En este libro, utilizando criterios y metodologia pedagógicos, se dan los fundamentos e instrumentos teórico-prácticos necesarios para el planteamiento de todo proyecto arquitectónico solar pasivo, de acuerdo con los principios éticos y económicos de conservación y ahorro de energia. Es decir: respeto de los presupuestos bioclimáticos, búsqueda de la máxima captación y acumulación de la radiación solar, y esmero en el aislamiento térmico de los cerramientos.

Un volumen encuadernado en cartulina ibiza plastificada, a cinco colores, de $16 \times 23 \mathrm{~cm}$, compuesto de 216 páginas, 217 figuras, 87 gráficos, 19 tablas y 10 cuadros.

Madrid, 1983. Precios: España 2.100 ptas.; 30 \$ USA. 